\title{
Propuesta de lineamientos normativos para el uso del suelo rural en el caso del cantón Palora - provincia de Morona Santiago.
}

\section{Proposal of normative guidelines for the use of rural soil in the case of canton Palora Morona Santiago province}

Vicente Patricio Quezada Ortega. ${ }^{1}$, Marco Benigno Avila Calle. ${ }^{2} \&$ Yonimiler Castillo Ortega. ${ }^{3}$

Recibido: 11-11-2020 / Revisado: 28-11-2020 /Aceptado: 05-12-2020/ Publicado: 02-01-2021

\begin{abstract}
.
DOI: https://doi.org/10.33262/cienciadigital.v5i1.1519

Enhancing the advantages of each territory is the main idea of endogenous development. For its practical realization, it will be essential the instrumentation of an adequate use and management of the soil, with an strong basis on Ecuadorian legislation. The case of the Palora canton, its agricultural activity and the sowing of pitahaya were analyzed in a specific way. It is necessary to highlight that this crop is the main source of the local economy, however, it has been implemented in a spontaneous and disorganized way, without having a minimum technical criterion of regulation in the use and management of the soil. The pitahaya cultivation has presented an unexpected growth, accentuated in the last five years, and continues increasing. This is configured as an economic bonanza that also constitutes a problem, since it puts at risk the fragile and rich cantonal biodiversity Sangay National Park, the abundant hydric resources and the cultural losses. Besides, it deepens different problems such us: change in the use of soil; the implementation of retreats towards roads and borders; the exaggerated soil fractioning; the opening of new roads; the intensive use of agrochemicals; and the contamination of natural resources like water and air. In order to know with certainty and in depth the reality of production in the area of study, the research has been developed using quantitative and qualitative methods, such us surveys and focus group interviews. The proposal for Palora's canton formulates a set of legal norms. Firstly, the current classification in urban

\footnotetext{
${ }^{1}$ Universidad, de Cuenca, Facultad de Arquitectura, Cuenca, Ecuador, pvquezada@hotmail.com https://orcid.org/0000-0002-7414-5742

${ }^{2}$ Universidad Católica, Facultad de Ingeniería y Arquitectura, Cuenca, Ecuador, mavila@ucacue.edu.ec http://orcid.org/0000-0002-2134-1432

3 Director de la Carrera de Economía en la Universidad Católica de Cuenca, ycastilloo@ucacue.edu.ec https://orcid.org/0000-0002-7710-5199
} 
soil determined by local regulations and rural soil was ratified. In the analysis of rural soil, it was sub-classified into four types of soil: productive, protection, extractive and buffer zones. Then, the intervention polygons were defined, and categories and subcategories were detail, everyone with their own areas of location at the parroquial level.

Keywords: Endogenous development, rural land use, normative guidelines / legal norms /local regulations

\section{Resumen:}

Potenciar las ventajas de cada territorio constituye la idea central del desarrollo endógeno. Para su concreción práctica resultará imprescindible la instrumentalización de un adecuado uso y gestión del suelo, en el que se tenga en cuenta como base el marco jurídico ecuatoriano. Se analizó el caso del cantón Palora, su actividad agrícola y la siembra de pitahaya de forma específica. Cabe aclarar que este cultivo constituye la principal fuente generadora de economía local. Sin embargo, esta se ha implantado de forma espontánea y desorganizada, sin contar con un mínimo criterio técnico de regulación en el uso y gestión del suelo. El cultivo de pitahaya presenta un crecimiento imprevisto, acentuado en los últimos cinco años y continúa incrementándose. Esto se configura como una bonanza económica que constituye también un problema, puesto que pone en riesgo la frágil y rica biodiversidad al ser parte del Parque Nacional Sangay, la abundante red hídrica y la perdida cultural. Además, profundiza problemas de diversa índole, por ejemplo: cambio de uso de suelo; implantación de retiros hacia vías y linderos; exagerado fraccionamiento del suelo; apertura de nuevas vías; intensivo uso de agroquímicos; contaminación de cuerpos hídricos y del aire. Con el fin de conocer con certeza y a profundidad la realidad de la producción en el área de estudio, la investigación se desarrolló usando métodos cuantitativos y cualitativos. Entre estas herramientas destaca la encuesta, entrevista y grupo focal, respectivamente. La propuesta planteada para el cantón Palora formula un conjunto de lineamientos normativos. En primera instancia, se ratificó la clasificación en suelo urbano determinado con ordenanza y suelo rural. en el análisis del suelo rural se ha subclasificado en cuatro tipos de suelos: productivo, protección, extractivo y amortiguamiento, de éstos se definió polígonos de intervención, categorías y subcategorías con sus respectivas áreas de localización a nivel de parroquias.

Palabras claves: Desarrollo endógeno, uso de suelo rural, lineamientos normativos.

\section{Introducción:}

En la actual coyuntura post COVID-19 resulta importante revalorar la realidad rural cuando de ella dependen los recursos naturales y, de igual forma, su base económica se estructura alrededor de la oferta ambiental en que se sustenta. Bajo esta perspectiva, aproximaciones como la gestión del suelo rural cobra mayor fuerza como instrumento para el ordenamiento territorial. No se puede dejar de mencionar que, en América Latina, uno de los temas más críticos en términos de desarrollo e inclusión son las enormes diferencias de pobreza y equidad entre zonas rurales y urbanas, es un aspecto que tiene un nivel de influencia mucho mayor que el espacio físico, el cual se convierte en el soporte o apoyo adicional de los grupos sociales y 
las comunidades.

El presente artículo busca una aproximación a las teorías del desarrollo y los principales enfoques planteados, los que se han orientado a resolver la situación de la pobreza o recuperación económica en diferentes realidades. Así, desde estos conceptos, se puede hacer un acercamiento a las ideas de lo local y lo endógeno como base de la propuesta de ordenamiento territorial rural y desarrollo económico. Por lo mismo, se plantea un análisis que permita ubicar los parámetros de un tema más concreto y aterrizado a la realidad rural, centrándose de manera específica en los enfoques del uso y gestión del suelo, para así proponer algunos lineamientos normativos.

Teorías sobre el desarrollo endógeno, el ordenamiento territorial rural y el uso de suelo. En el marco de las teorías clásicas, reflexiona Martínez-Echevarría (2011), se puede asegurar que siempre se ha generado una base de pensamiento que busca "mejorar los niveles de vida para alcanzar la felicidad" (p. 83), desde el mismo pensamiento aristotélico. Al respecto, Iturralde (2019) propuso una aproximación y análisis sobre la evolución del concepto de desarrollo desde la postura económica hasta la multidisciplinaria. El autor se encarga de describir las diferentes etapas y propuestas que se sintetizan así: Teoría de la Modernización, Estructuralista, Neoclásica Institucional, Neomarxista, Neoliberal, Desarrollo Sostenible, Crecimiento a Escala Humana, Desarrollo Humano Sostenible. De estas principales teorías del desarrollo se desprende toda la elaboración conceptual que predominó en las décadas desde los años cincuenta hasta finales de los ochenta. De este modo, se intenta superar el reduccionismo economicista, por lo que se replantea una nueva posición que incorpora la visión a enfoques más amplios como los problemas sociales y ambientales.

No se puede dejar a un lado que desde la Segunda Guerra Mundial y durante más de cuarenta años, los resultados alcanzados fueron pocos. Estos dos conceptos mencionados predominaron en las acciones de las instituciones y organismos internacionales que se ocupaban del desarrollo. Sin embargo, las críticas provocaron momentos y argumentos que ligaron el desarrollo con lo endógeno. Vásquez (2007) planteó que "es imperativo repensar el desarrollo desde otras corrientes, donde las interpretaciones confluyan en diferentes visiones del desarrollo, pero que compartan una misma lógica teórica y, sus lineamientos confluyan en las políticas de desarrollo que generen un verdadero estado de bienestar" (p. 206). Este planteamiento se puede relacionar con el análisis de Gómez (2014), quien concluyó lo siguiente:

"El concepto de desarrollo endógeno aparece en un primer momento como el intento de relacionar una concepción del desarrollo entre localidades y territorios rezagados, en un segundo momento, se habla de un desarrollo endógeno, a partir de la inclusión de formas más flexibles de organización territorial de la producción" (p. 119)

Sin duda, hoy en día el desarrollo tiene una mirada fija en el territorio y sus potencialidades; una perspectiva que tiene un enfoque multidisciplinario.

Desde lo multidisciplinario es fundamental precisar que el desarrollo endógeno es un concepto empleado por autores de distintos campos de las ciencias sociales y actores públicos 
(ciudadanos, dirigentes y autoridades); sujetos que poseen ideologías diferentes y, de ahí, que se emplee ese concepto con significados y objetivos muy diversos. Los autores Gil et al (2018) añadieron que "existen dos vertientes sobre el desarrollo, tomando en cuenta que ese término no es exclusivo de la economía, se puede aplicar a los procesos sociales, esas vertientes son el desarrollo como un fin y el desarrollo como una estrategia" (p. 15). Sin duda, un nobel debate que toma como fundamento y enfoque lo territorial y que, a su vez, busca transformaciones en la economía y la sociedad como consecuencia del aumento de la integración económica, política, ambiental y cultural. En correspondencia con esto, Torres et al (2011) reafirmó que "hoy en día, el desarrollo es algo más que la apuesta por el crecimiento económico como soporte de la riqueza social y el bienestar humano" (p. 1).

Estos enfoques endógenos han surgido a consecuencia de profundizar el principio rector del desarrollo. En la práctica, se reclama dar mayor peso a otros aspectos igualmente importantes como es el caso de la relación con la naturaleza, el incremento de capacidades locales, la sustentabilidad de las intervenciones, el territorio como base de la economía.

Para Valdizán (2007), Dollfus (1997), Franco (2013), Hoyuela y Citores (2007), el desarrollo endógeno está ligado a soluciones de problemas en pequeña escala y a su realidad particular y vivencial, ecológica, comunitaria y de época. Por lo que encuentra un mayor empuje en el plano microeconómico "en busca del agente principal del desarrollo, puesto que la dinámica de la realidad zonal depende de la integración y actuación del emprendedor local en el territorio" (Barroso y Flores, 2010, p. 46). En esta línea Perroux (1991) aporta al concepto y define el desarrollo endógeno "como la combinación de los cambios mentales y sociales de una población que la vuelven apta a hacer crecer, cumulativa y durablemente su producto real global" (p. 191).

"La construcción planificada del territorio es la esencia de la ordenación territorial, cuyo objetivo primordial consiste en ir construyendo un sistema" (Gómez y Gómez, 2014, p. 5) que busca resolver los conflictos en beneficio del interés común. Los autores Gómez y Gómez (2014) confirmaron que es tan importante como "lo que se debe hacer es lo que no se debe hacer, y donde tanto o más importante que resolver los problemas actuales es prevenir los potenciales" (p. 5).

La construcción del desarrollo desde la perspectiva endógena se puede concretar desde la ley orgánica de ordenamiento territorial uso y gestión del suelo LOOGTUS (2016), conceptualizó el término de la siguiente forma:

"El suelo es el soporte físico de las actividades que la población lleva a cabo en búsqueda de su desarrollo integral sostenible y en el que se materializan las decisiones y estrategias territoriales, de acuerdo con las dimensiones social, económica, cultural y ambiental" (p.8)

Para la Organización de las Naciones Unidas [ONU] (2018) el Plan de Uso y Gestión de Suelo "constituye un instrumento normativo de planificación, paralelo al PDOT, que permite a los Gobiernos locales regular y gestionar el uso, la ocupación, el fraccionamiento y transformación 
del suelo, conforme la visión de desarrollo y el modelo territorial deseado" (p.24).

\section{Marco jurídico - legal y normativa técnica para el ordenamiento territorial rural ecuatoriano.}

La presente investigación científica se ejecuta dentro de un marco jurídico o conjunto de leyes y normativas vigentes en el Ecuador. Estas se centran en torno al ordenamiento territorial y, de forma concreta, en el uso de suelo rural. A continuación, se analiza la jerarquía de la pirámide kelseniana:

La Constitución de la República del Ecuador [CRE] (2008) establece entre las competencias exclusivas de los Gobiernos Autónomos Descentralizados (GAD) la planificación y el desarrollo y, de igual forma, la formulación de los correspondientes planes de ordenamiento territorial de sus circunscripciones. En estas se dispone explícitamente para los gobiernos autónomos descentralizados cantonales el objetivo específico de "regular el uso y ocupación del suelo urbano y rural" (Presidencia de la República del Ecuador, 2010, art. 55).

El Código Orgánico de Organización Territorial, Autonomía y Descentralización [COOTAD] (2010) establece en varios artículos las funciones y competencias exclusivas de cada nivel de GAD, así como las responsabilidades de cada autoridad. Asimismo, resalta la obligatoriedad de la planificación territorial rural. En el art. 57 se establece que se debe "regular y controlar, mediante la normativa cantonal correspondiente, el uso del suelo en el territorio del cantón, de conformidad con las leyes sobre la materia, y establecer el régimen urbanístico de la tierra" (Presidencia de la República del Ecuador, 2010a).

El Código Orgánico de Planificación y Finanzas Públicas [COPYFP] (2010) define los ámbitos de la planificación en los que se determinan los contenidos de esta. En el art. 44 se estipulan:

“[...] Los planes de ordenamiento territorial de los gobiernos autónomos descentralizados observarán los siguientes criterios: [...] corresponde exclusivamente a los gobiernos municipales y metropolitanos la regulación, control y sanción respecto del uso y ocupación del suelo en el territorio del cantón." (Presidencia de la República del Ecuador, 2010b)

El Código Orgánico del Ambiente [COA] (2017) es un conjunto de normas garantes de la armonía con la naturaleza, plantea como objeto:

"Garantizar el derecho de las personas a vivir en un ambiente sano y ecológicamente equilibrado, así como proteger los derechos de la naturaleza para la realización del buen vivir o sumak kawsay [además resalta] las Facultades de los Gobiernos Autónomos Descentralizados Metropolitanos y Municipales en materia ambiental [...]" (art. 1)

La Ley Orgánica de Ordenamiento Territorial, Uso y Gestión del Suelo [LOOTUGS ] (2016) tiene por objeto:

“[...] Fijar los principios y reglas generales que rigen el ejercicio de las competencias de ordenamiento territorial, uso y gestión del suelo urbano y rural, y su relación con otras que incidan significativamente sobre el territorio o lo ocupen, para que se articulen eficazmente, promuevan el desarrollo equitativo y equilibrado del territorio y propicien 
el ejercicio del derecho a la ciudad, al hábitat seguro y saludable." (art. 1)

El Reglamento a la Ley Orgánica de Ordenamiento Territorial, Uso y Gestión del Suelo (2019) determinan para el Plan de Uso y Gestión del Suelo los Contenidos del componente estructurante y los contenidos mínimos del componente urbanístico. En el art. 11 se define "el Plan de Uso y Gestión del Suelo será aprobado mediante la misma ordenanza municipal o Metropolitana que contiene el Plan de Desarrollo y Ordenamiento Territorial Cantonal [...]" (Decreto 680, 2010) en el procedimiento de aprobación.

La Norma Técnica de contenidos mínimos, procedimiento básico de aprobación y proceso de registro formal de los planes de uso y gestión de suelo y, los planes urbanísticos complementarios de los gobiernos autónomos descentralizados municipales y metropolitanos (Consejo Técnico de Uso y Gestión del Suelo, 2020). Esta normativa se convierte en un instrumento metodológico y de detalle técnico, la cual se encarga de determinar los contenidos mínimos, el procedimiento básico de aprobación y el proceso de registro formal de los planes de uso y gestión del suelo a ser expedidos por los gobiernos autónomos descentralizados municipales.

El marco jurídico ecuatoriano evidencia una abundante normativa. Sin embargo, Pauta-Calle (2019) evidencia "Se ha creado una superposición de determinaciones con aquellas que el ordenamiento territorial asigna a un instrumento que por mandato constitucional debe regular el uso y la ocupación del suelo"; Reflexión que no limita su aplicación.

\section{Metodología:}

En cuanto al levantamiento de información relevante para el diagnóstico del presente artículo se utilizaron las técnicas de investigación cualitativas y cuantitativas.

Para la recopilación de información cualitativa se aplicaron dos técnicas: 1) dos entrevistas grupales semiestructuradas con los tres técnicos municipales que laboran en la "mesa de la pitahaya". La información fue recopilada mediante grabaciones de audio y, a su vez, se elaboraron informes que contienen los principales resultados y conclusiones. 2) Se desarrolló un grupo focal con productores de pitahaya de Palora. En total, se eligieron ocho participantes que reflejan la realidad del universo. El grupo se conformó por hombres mestizos y shuar considerando la proporción étnica cantonal. Además, asistió un representante de las asociaciones de productores. La información del grupo focal se registró en grabaciones de video y los informes fueron procesados y estructurados para establecer las variables y temática del cuestionario a ser aplicado en la encuesta.

La información cuantitativa se aplica para obtener datos actualizados de la situación económica-productiva de Palora. Para el efecto se diseñó una encuesta que permitió recabar la mayor cantidad de datos descriptivos de las prácticas agrícolas, a través de los siguientes parámetros que permitan tener una lectura completa de la actividad antrópica en las UPAs: 
1. Localización de la finca y datos del informante;

2. Datos de la unidad productiva y del nivel de actividad;

3. La producción agrícola y la comercialización de los productos;

4. Cambios en el uso del suelo;

5. Generación de ingresos y de bienes para el autoconsumo de la unidad productiva;

6. Características socioeconómicas;

7. Potenciales desarrollos agroproductivos de expansión y de conversión;

Con el fin de determinar el tamaño de la muestra se usó el concepto de población finita, dado que en este caso el tamaño del universo es conocido.

Si la población es finita, es decir, si se conoce el total de la población (N) y, a su vez, se desea saber el tamaño de la muestra (n), cuántos individuos del total se deben estudiar, se debe estudiar (n) para poder inferir en la población. En tal caso, la respuesta sería:

$$
\mathrm{n}=\frac{N * Z_{\alpha}^{2} p * q}{d^{2} *(N-1)+Z_{\alpha}^{2} * p * q}
$$

A continuación, se explican sus respectivas cifras:

- $\quad \mathrm{N}$ : total de la población.

- $Z_{\alpha}^{2}: 1,96^{2}$ (si la seguridad es del $95 \%$ ).

- p: proporción esperada (en este caso el $5 \%=0,05$ ).

- q: 1 -p (en este caso 1-0,05=0,95).

- d: precisión (en este caso se requiere $3 \%$ ).

Teniendo en cuenta que el universo de análisis se corresponde con el número de predios rurales existentes en el cantón Palora, de la información recibida de la Dirección de Planificación del [GAD] Municipal se tienen 3.232 UPA. Cabe aclarar que se espera que el diseño muestral tenga significancia estadística a nivel parroquial sobre la base del número de las UPA, identificadas a nivel parroquial.

En total, se definió el levantamiento de 322 encuestas, de las cuales el $19 \%$ corresponden a Arapicos, el $21 \%$ a Palora, el $20 \%$ a Cumandá, el $21 \%$ a Sangay y el $19 \%$ a 16 de agosto. La aplicación de la encuesta y su procesamiento se realizó en el mes de octubre del 2020. Para el efecto se contó con un equipo de cinco encuestadores y un supervisor de campo, oriundos de Palora. Esta situación facilitó el proceso de interacción con los finqueros y, asimismo, permitió la recolección oportuna de los datos. La base de datos y el procesamiento de la información recopilada se analizó mediante el programa SPSS, lo que permitió obtener datos específicos para el diagnóstico; y en la fase de propuesta determinar lineamientos normativos que aseguren la mejor utilización de las potencialidades y capacidad de acogida del territorio en función de un desarrollo armónico, sustentable y sostenible del suelo rural del cantón Palora.

Para formular la propuesta se utilizó información cartográfica principalmente del Ministerio del Ambiente: topografía, hidrografía, zonas de riesgos, áreas protegidas, vialidad, concesiones 
mineras, mecánica del suelo. La que posibilitó la creación de mapas temáticos y cuadros que permiten la determinación de polígonos y de manera específica las categorías y subcategorías según las potencialidades y realidades particulares del territorio.

\section{Diagnóstico del uso y gestión del suelo rural del cantón Palora.}

El cantón Palora encuentra su origen en la ley de Reforma Agraria y Colonización de la Región Amazónica en el año de 1962. Dicha ley trajo consigo la inmigración de personas que arribaron en calidad de trabajadores de la compañía del té, luego de explorar las tierras y darse cuenta de la fecundidad de estas para la agricultura y ganadería. Poco a poco, se convirtieron en propietarios finqueros, es decir, colonos.

El presente diagnóstico resume los principales problemas de las actividades productivas rurales del cantón Palora, en los aspectos sociales, económicos productivos, morfológicos territoriales, y urbanísticos. Para ponderarlos con la capacidad de uso de la tierra, ecosistemas frágiles, zonas de amenaza y áreas patrimoniales, criterios que permitan definir lineamientos normativos sobre la base de las potencialidades y particularidades de cada área: clasificación, subclasificación, categorías y subcategorías del uso de suelo rural.

Un antecedente importante se halla en el Plan de Desarrollo y Ordenamiento Territorial Cantonal del año 2015, el cual resalta la cobertura de uso de suelo rural. "Predominan cultivos de yuca, plátano, papa china, naranjilla y en menor proporción caña de azúcar, además, existen cultivos de té y en menor escala pitahaya, productos que se exportan; pero el recurso más explotado por la población es la madera".

Según la página web del Gobierno Autónomo Descentralizado Municipal, el territorio cantonal de Palora comprende 145670 hectáreas. En esta extensión predomina la clasificación de suelo rural en un $99.5 \%$ y aproximadamente el 50\% del territorio cantonal se categoriza como área protegida, la cual pertenece al Parque Nacional Sangay con 70000 hectáreas. Adicional a esto, se identifican extensiones de bosques con vegetación arbustiva, vegetación herbácea, áreas de pastizales y cultivos, páramo y, desde luego, plantaciones forestales.

El suelo rural ha sufrido un cambio muy significativo, debido al uso agrícola extensivo e intensivo por la proliferación de plantaciones de pitahaya. En la actualidad existen alrededor de 2043,91 hectáreas de pitahaya sembradas en estos suelos, lo que ha generado varios problemas, entre ellos: fraccionamientos ilegales del suelo rural con superficies mínimas de entre media y tres hectáreas. Estas divisiones se corroboran con los datos técnicos fraccionamientos realizados durante los años 2015 hasta enero del 2020, confirmándose un total de 545. De acuerdo con esta información, entre 0 hasta 3 hectáreas corresponde a 341 fraccionamientos.

Otro de los fenómenos que genera el fraccionamiento del suelo rural consiste en que produce 
apertura no planificada y espontánea de nuevas vías. Es decir, incrementa la extensión de la red vial que requiere mantenimiento y, a consecuencia de los efectos del clima, presenta un rápido deterioro. $\mathrm{Al}$ mismo tiempo, se construye un sistema vial laberintico y desorganizado. Las históricas fincas de 50 hectáreas al ser fraccionadas buscan una mayor ganancia en la venta del suelo. De esto resultan pequeñas parcelas, las cuales son sembradas con pitahaya. El tamaño de las Unidades Productivas Agrícolas (UPA) oscila en el 35,6 \% con tres hectáreas o menos, el 26,6 \% entre 4 y 10 hectáreas, el 23,9 \% entre 15 y 45 hectáreas y, por último, el 13,9\% con la extensión original de 50 o más hectáreas.

El cantón Palora se localiza dentro de la rica red hidrográfica de la "cuenca del río Pastaza" (Yépez, 2015, p. 5), conformada por cuatro subcuencas: drenajes menores, río Chambo, río Llushín y río Palora. Asimismo, cuenta con 21 microcuencas en donde los ríos Pastaza y Palora son los que poseen mayor recorrido. Cabe recordar que allí afloran los siguientes ríos: Encanto, Cuyuimi, Llushín, Chuya Llushín, Amúndalo, Metzeras, Numbaime, Nayanamaca. Esta red hidrográfica influye directamente en el abastecimiento de agua hacia cada una de las parroquias del Cantón. De los nombrados con anterioridad, el río Metzeras, el cual cruza la zona urbana de la cabecera cantonal de Palora y del río Nayanamaca, se capta el agua, que luego de pasar por la planta de tratamiento, se distribuye para consumo humano en la ciudad de Palora. Por lo tanto, la abundancia de todos estos afluentes naturales como ríos, esteros y quebradas necesitan ser protegidos.

En todo el cantón Palora se han determinado dos características de riesgos: inundaciones y movimientos en masa. Existe un total de 5174 hectáreas en riesgo de inundación, las cuales se presentan mayormente a zonas con plantaciones de pitahaya. Además, es importante tomar en cuenta que las zonas inundables se ubican en relieves menores a los 710 m.s.n.m. y, cuentan con suelos de baja permeabilidad. Lo anterior genera ambientes que propician inundaciones. El riesgo de movimientos en masa en el cantón se manifiesta en un total de 5176,13 hectáreas, las cuales están ubicadas en su mayoría en zonas pobladas y cultivos de pitahaya. La categoría de alto riesgo se ubica en dos puntos importantes que corresponden a los puentes sobre el río Pastaza. Además, pertenecen a zonas urbanas y centros poblados.

A pesar de la importancia de la actividad agrícola en el cantón Palora, el desarrollo de estas no ha tenido orientación ni control desde las instancias o niveles de gobierno. En otras palabras, la falta de regulación que determina el uso y gestión del suelo rural es la que ha generado cambios sustanciales en la ampliación de la frontera agrícola, cambio de uso y concentración del monocultivo. La irrupción masiva del cultivo de la pitahaya ha tenido un crecimiento importante durante los últimos años; esta cifra ha pasado de producir 35 toneladas en el año 2000 a generar alrededor de 6 mil toneladas de la fruta en el 2019.

La preocupación central radica en la tenencia de la tierra rural, dado que el 68,6 \% de los propietarios tienen su origen en otros cantones de la provincia, otras provincias del país e, incluso, en otras naciones. En otras palabras, significa que la riqueza económica de la producción de pitahaya no se queda en el Cantón, aunque los efectos de contaminación sí 
permanecerán por algunas décadas.

El producto que genera el mayor ingreso de las UPA es la pitahaya con un 64,3\%, seguido por el 18,6 \% con ninguno, mientras que el 3,9 \% es el ganado. Enseguida, el $3 \%$ es la caña, el 2,7 $\%$ la naranjilla y el restante $7,8 \%$ mencionan al café, cítricos, guanábana, orito, plátano, tilapia, yuca o madera como lo más rentables. La importancia de la pitahaya se evidencia al mantenerse como el principal cultivo permanente con el $73 \%$ de los productores. En contraste, el 8,4\% disponen de una diversidad de cultivos tales como el café, canela, caña, guaba, guanábana, guayaba, limón, naranjilla, orito y té.

En cuanto al tamaño de los lotes de los cultivos permanentes, el $60 \%$ son de una hectárea o menos, por el alto costo de implantación de la pitahaya, y al tiempo de espera para el inicio de la producción, esto es dos años. El 17,4 \% de los cultivos tiene entre 1 y 2 hectáreas; el $6 \%$ entre 2 y 3 hectáreas y, finalmente, el 7, 8\% de las plantaciones tienen entre 5 y 10 hectáreas. Si se analizan las plantaciones por los años transcurridos desde la siembra, se encuentra que el 13,3 \% está recién sembrada. El 4,6\% tiene menos de 1 año, el 13,8 \% menos de 2, mientras que el 68,22 \% alude a tres años o más.

En lo referente a la conservación el $72 \%$ de los productores agrícolas, sí realizan prácticas para la preservación del suelo y el agua. Entre las principales prácticas que se ejecutan en las unidades productivas del cantón están: las zanjas que son utilizadas por el 70,2\% de los productores; las barreras vivas por el $17 \%$ de los productores; el barbecho por el 5,5\% de los productores, así como otras actividades menores tales como las terrazas, uso de material orgánico, la rotación de cultivos y la cobertura vegetal.

En cuanto a la naturaleza de la producción de la pitahaya esta demanda la intensiva utilización de fertilizantes, abonos, control de maleza, insecticidas, fungicidas, material foliar y otros plaguicidas. Por lo que resulta de vital importancia establecer las prácticas y el uso de los diferentes productos disponibles en el mercado. Con los productores se pudo establecer cuáles son los productos más empleados en el ciclo productivo, así como el listado completo de los productos disponibles que son utilizados con menor frecuencia.

Una de las etapas críticas de la producción de bienes agrícolas se fundamenta en la comercialización, ya que el estado de los bienes que se comercializan influirá en los precios que se adquieran por estos. En este caso, solo el $2 \%$ de la producción se comercializa en estado seco, mientras que el $98 \%$ se lo distribuye como fruta fresca.

Los canales de comercialización utilizados en Palora para los productos permanentes son varios. El más utilizado es el de la venta al centro de acopio, al cual se destina el 63,5\% de la producción. En contraste, los intermediarios canalizan el 20,3 \% de la producción, las asociaciones el 7,3\% y los consumidores el $8 \%$. Respecto a los efectos del fraccionamiento de suelo en el cultivo, solo el $3 \%$ de las unidades productivas observaron cambios en las áreas de cultivo. En este mismo contexto, frente a si el fraccionamiento permitió el cambio a otros 
cultivos, este se cumplió en el $3 \%$ de las unidades fraccionadas.

\section{Resultados y discusión}

La construcción de la propuesta se estructura al sobreponer información de dos fuentes que confluyen en la presente investigación; por un lado los resultados obtenidos en la encuesta, y por otro la cartografía oficial del Ministerio del Ambiente Ecuador (MAE), en temas como topografía, hidrografía, zonas de riesgos, áreas protegidas, vialidad, concesiones mineras, mecánica del suelo, generando un debate desde la teoría del desarrollo endógeno para determinar un conjunto de lineamientos normativos que orienten la expansión, control y regulación del cultivo de pitahaya.

\section{Propuesta de lineamientos normativos para la clasificación del uso de suelo rural del cantón Palora.}

Los lineamientos normativos son las disposiciones emitidas por un gobierno sea local o nacional que buscan generar acciones que den respuestas a las diversas demandas y/o preocupaciones de la colectividad. Algunos autores enfatizan en el tema de la ciudadanía, por ejemplo, Ruiz y Cadénas (s.f.) conceptualizaron las políticas públicas como "el conjunto de actividades de las instituciones de gobierno, actuando directamente o a través de agentes, y que van dirigidas a tener una influencia determinada sobre la vida de los ciudadanos" (párr. 25). También Pallares (1988), como se citó en Ruiz y Cadénas (s.f.), afirmó que estos conceptos deben ser considerados como "procesos decisionales, un proceso de decisiones que se llevan a cabo a lo largo de un plazo de tiempo" (párr. 25). Los autores mencionados ayudan a tener claridad del concepto de formulación de políticas públicas y de su parte operativa. La formulación de políticas públicas, según Rodríguez (s.f.), expuso que implica previamente considerar algunas preguntas: ¿cómo podemos intervenir? ¿qué herramientas están a nuestro alcance?; preguntas que serán respondidas para dar forma a nuestro planteo epistemológico y ontológico del mundo y de las ciencias sociales, en particular la ciencia política.

Una interesante reflexión sobre la articulación conceptual del ordenamiento territorial y decisiones públicas fue planteada Gómez y Gómez (2014), quienes concluyeron que "frente a la indeseable evolución tendencial se plantea la evolución planificada del sistema territorial, que adecuadamente gestionada, lleva a un sistema territorial satisfactorio, y donde tanto o más importante que resolver los problemas actuales es prevenir los potenciales" (p. 5).

Complementan las nuevas nociones de la planificación Sánchez y Gutiérrez (2013) al plantear: "el ordenamiento territorial ha tenido como principios reguladores, la función social y ecológica de la propiedad, la prevalencia del interés general sobre el particular y la distribución equitativa de las cargas y de los beneficios" (p. 43). Las definiciones de los usos del suelo es una responsabilidad pública y privada. Estas deben ser pensadas desde la redefinición social y ambiental, necesariamente enfatizadas desde una visión prospectiva.

Lo ratifican Samper y Torrens (2015) al plantear "la construcción de las políticas públicas para 
el desarrollo de los territorios rurales es un proceso social complejo donde interactúan múltiples fuerzas sociales y político-institucionales. Estas, se relacionan interactivamente con los distintos tipos de actores en los territorios" (p. 1). En este sentido es importante que las políticas y programas nacionales responden a las dinámicas propias de cada país y de sus territorios subnacionales.

Siguiendo lo planteado en la LOOGTUS, en el cantón Palora se encuentra claramente determinada la forma de clasificación del suelo: en suelo urbano, áreas de centros parroquiales poblados, y suelo rural, que es el saldo territorial, en el caso de Palora comprende el $99.5 \%$ del territorio total. A partir de esta realidad, se realiza la sub-clasificación del suelo rural, para ello la mencionada determina cuatro tipos.

\begin{tabular}{llc}
\hline SUBCLASIFICACIÓN & AREA (HA) & $\mathbf{( \% )}$ \\
\hline PRODUCCIÓN & 65946.01 & 45.45 \\
PROTECCIÓN & 78506.55 & 54.10 \\
EXPANSIÓN URBANA & 411.78 & 0.28 \\
APROVECHAMIENTO EXTRACTIVO & 243.92 & 0.17 \\
TOTAL & $\mathbf{1 4 5 1 0 8 . 2 6}$ & $\mathbf{1 0 0}$ \\
\hline
\end{tabular}

Tabla 1. Sub-clasificación del suelo rural

Fuente: elaboración propia

Los lineamientos normativos para el uso de suelo se proponen a nivel de cada parroquia. A partir de la subclasificación se definen polígonos de intervención con su respectiva categoría y subcategoría, en la que se determina la extensión en áreas.

\section{Propuesta de lineamientos normativos de uso de suelo productivo:}

Para el Suelo Rural del cantón Palora se han determinado 4 categorías dentro de los Polígonos de Intervención Territorial, en base al análisis cartográfico de los territorios con la Subclasificación de Producción:

Categoría B1.- con una pendiente de hasta 70\%; profundidad efectiva del suelo mayor a $50 \mathrm{~cm}$; textura, salinidad, toxicidad, fertilidad, y drenaje indiferenciadas; pedregosidad poco frecuente. Esta categoría representa el $1.02 \%$ del territorio. Las propiedades de esta categoría del suelo, restringen el cultivo a sembríos endémicos y combinados, con restricciones de uso y densidades no mayores al $60 \%$ del área total de cada lote.

Categoría B2.- con pendientes de hasta el $100 \%$; profundidad mayor a $20 \mathrm{~cm}$; textura, salinidad, toxicidad, fertilidad, y drenaje natural indiferenciados, pedregosidad poco frecuente. Constituye el $13.78 \%$ del suelo cantonal. Las particularidades descritas determinan una prohibición total de todo tipo de cultivos.

Categoría P0.- Se identifica una pendiente de hasta el 40\%; profundidad efectiva del suelo mayor a $50 \mathrm{~cm}$, textura superficial, salinidad, fertilidad, drenaje natural y régimen de humedad 
ISSN: 2602-8085

de suelo indiferenciadas; pedregosidad de nula hasta frecuente; toxicidad de nula hasta ligera; duración de inundación hasta 9 meses. Representa el 26.58\% del suelo cantonal.

Las características descritas en esta categoría de suelo, posibilitan el emplazamiento de nuevos sembríos de pitajaya, restringiendo su densidad de uso a un $60 \%$ del área total de cada lote.

Categoría S0: Incluyen tierras con severas limitaciones físicas, que no permitirán ningún uso agropecuario, donde debe mantenerse la cobertura vegetal natural existente, por razones de conservación de los recursos.

Se establece en pendientes mayores al 100\%; duración de inundación mayor a 9 meses; y profundidad efectiva, textura superficial, pedregosidad, salinidad, toxicidad, fertilidad, drenaje natural, régimen de humedad de suelo y régimen de temperatura del suelo indiferenciados. Conforma el $3.92 \%$ del área cantonal.

\begin{tabular}{|c|c|c|}
\hline $\begin{array}{c}\text { CATEGORÍA } \\
\text { PRODUCCIÓN }\end{array}$ & $\begin{array}{c}\text { AREA } \\
(\text { Ha) }\end{array}$ & $\begin{array}{c}\text { PORCENTAJE } \\
(\boldsymbol{\%})\end{array}$ \\
\hline B1 & 1484.18 & 1.17 \\
\hline B2 & 19989.87 & 13.78 \\
\hline P0 & 38568.65 & 26.58 \\
\hline S0 & 5681.50 & 3.92 \\
\hline TOTAL & $\mathbf{6 5 7 2 4 . 1 9}$ & $\mathbf{4 5 . 4 5}$ \\
\hline
\end{tabular}

Tabla 2. Categorías de producción

Fuente: elaboración propia con base en el Ministerio del Ambiente

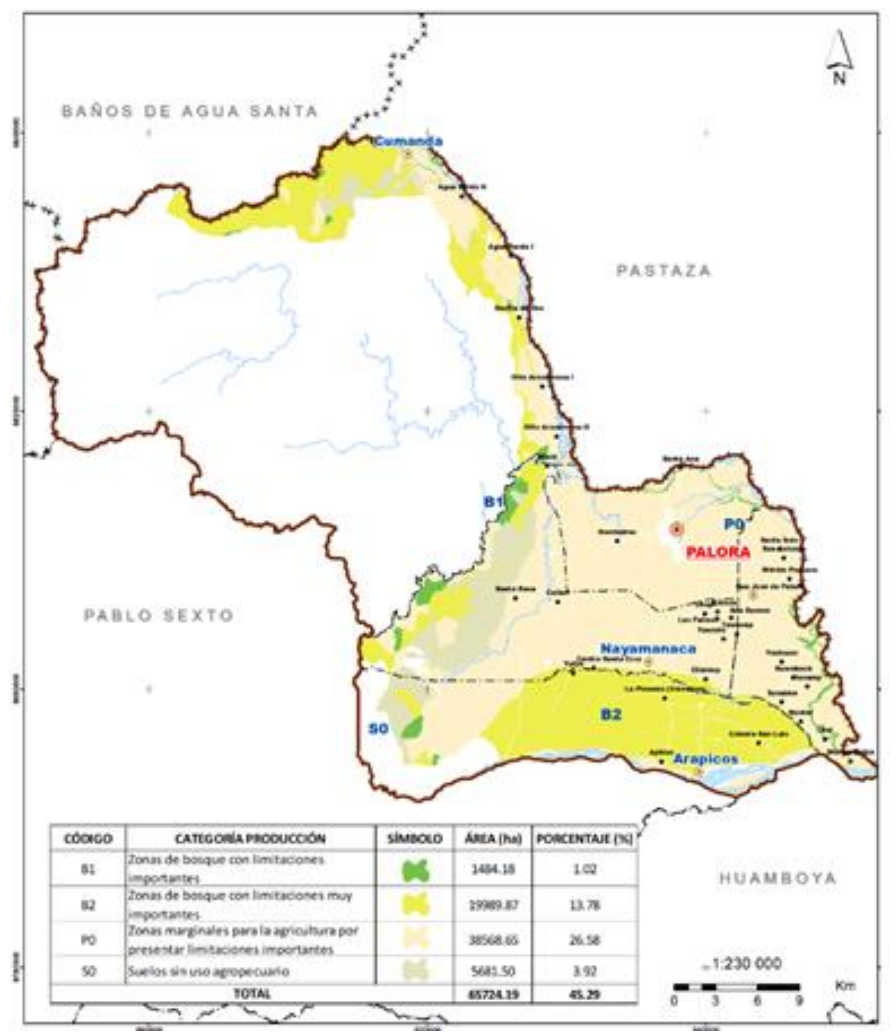

Mapa 1. Categorías de producción del cantón Palora Fuente: elaboración propia con base en el Ministerio del Ambiente

Cada una de las categorías establecidas, se determinan las subcategorías de los polígonos de intervención de producción sujetas a limitaciones que afectan a la 
aptitud de las tierras, las cuales están determinadas de acuerdo con la naturaleza del suelo. El subíndice "a" define un suelo sin limitaciones, el subíndice "b"; suelo con ligeras limitaciones, subíndice "c"; suelo con importantes limitaciones, finalmente el subíndice "d"; determina suelos con muy importantes limitaciones.

\begin{tabular}{|c|c|c|}
\hline $\begin{array}{l}\text { SUBCATEGORÍA } \\
\text { PRODUCCIÓN }\end{array}$ & $\begin{array}{c}\text { AREA } \\
(\mathbf{H a})\end{array}$ & $\begin{array}{c}\text { PORCENTAJE } \\
(\mathbf{\%})\end{array}$ \\
\hline $\mathrm{B} 1 \mathrm{~b}$ & 15.55 & 0.18 \\
\hline $\mathrm{B} 1 \mathrm{~d}$ & 1468.63 & 1.01 \\
\hline $\mathrm{B} 2 \mathrm{c}$ & 356.46 & 0.25 \\
\hline $\mathrm{B} 2 \mathrm{~d}$ & 19633.41 & 13.53 \\
\hline $\mathrm{Pa}$ & 135.63 & 0.09 \\
\hline $\mathrm{Pb}$ & 12157.28 & 8.38 \\
\hline $\mathrm{Pc}$ & 23763.29 & 16.38 \\
\hline $\mathrm{Pd}$ & 2512.45 & 1.73 \\
\hline $\mathrm{Sc}$ & 5681.50 & 3.92 \\
\hline TOTAL & $\mathbf{6 5 7 2 4 . 1 9}$ & $\mathbf{4 5 . 4 5}$ \\
\hline
\end{tabular}

Tabla 3. Subcategorías del suelo rural Fuente: elaboración propia

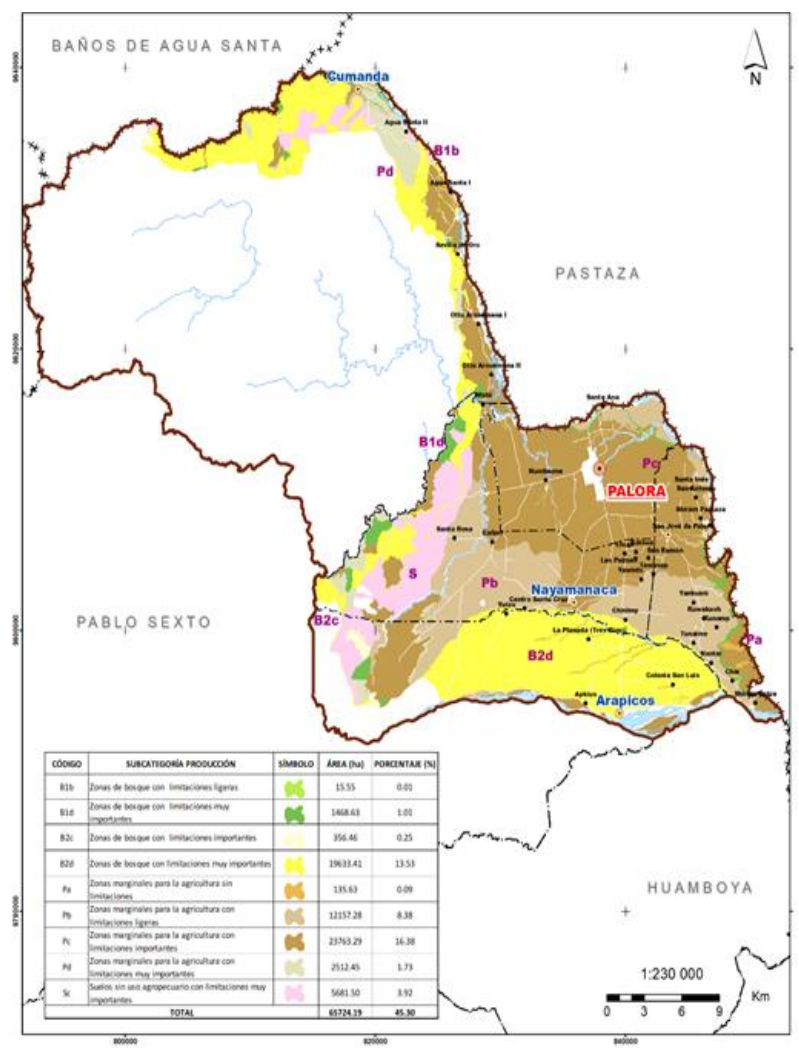

Mapa 2. Subcategorías del suelo rural Fuente: elaboración propia con base en el Ministerio del Ambiente

\section{Propuesta de lineamientos normativos para fraccionamiento de suelo productivo:}

Hacia las vías rurales Palora-Numbaime, Palora-Mojón-San José, Mojón-Sangay- Arapicos existentes, se establecerá una franja que comprende $200 \mathrm{~m}$ desde la línea de fábrica del lote a cada lado de la vía. Sobre este suelo rural se permite el fraccionamiento mínimo de 1 hectárea. Se establece una zona de amortiguamiento en todo el límite interno del Parque Nacional Sangay, con las propiedades del cantón Palora, donde no se permitirá el fraccionamiento menor a 5 hectáreas. En el resto del territorio productivo se permitirá un fraccionamiento de 2 hectáreas.

En las zonas periféricas a los centros urbanos se ha establecido un área de amortiguamiento con un radio de influencia de $500 \mathrm{~m}$ para las cabeceras cantonal y parroquiales urbanas. En los centros poblados urbanos se determinará una franja de $500 \mathrm{~m}$, donde no se permitirá sembrío de monocultivo y el uso de productos químicos.

\section{Propuesta de lineamientos normativos de protección ecológica:}

En el suelo rural del cantón Palora, Subclasificado como de Protección se estructura en tres polígonos: 1.- áreas de riesgo que comprende la categoría de procesos geodinámicos o alteración de la corteza terrestre, y se orientan a su recuperación 2.- Suelos de Patrimonio Natural que comprende categoría de protección hídrica, importancia hídrica y representación hídrica, 3.- Suelos de protección de márgenes de vías. Para cada categoría se enfatiza la 
intervención, en los apartados siguientes.

\section{Propuesta de lineamientos normativos de áreas de riesgo:}

El área de riesgo del cantón Palora, está determinada por el municipio, aquí se localizan los eventos naturales de riesgos y amenazas más recurrentes, como: los movimientos en masa se ubica básicamente la parroquia Arapicos, con una extensión de 150 hectáreas es decir 0,10\% del territorio cantonal, se ha planteado la normativa de restricción absoluta de uso y ocupación de dicho territorio, al tiempo de proponer zona de utilidad pública y reubicación de quienes aquí habitan.

\section{Propuesta de lineamientos normativos de protección hídrica:}

En las márgenes de protección de ríos, quebradas y esteros se formula la normativa definida como protección ecológica y, asimismo, se determina que el suelo se dejará para establecer los proyectos de equipamientos recreativos. Todo predio limitado o atravesado por un río mantendrá una franja de protección de acuerdo al ancho del río. Se tendrán los siguientes rangos:

$0-5 \mathrm{~m}$ retiro desde el borde o ribera del río $5 \mathrm{~m}$.

5 - $10 \mathrm{~m}$ retiro desde el borde o ribera del río $10 \mathrm{~m}$.

10 - $15 \mathrm{~m}$ retiro desde el borde o ribera del río de $15 \mathrm{~m}$.

15 - $30 \mathrm{~m}$ retiro desde el borde o ribera del río de $30 \mathrm{~m}$.

Mayores a $30 \mathrm{~m}$ retiro desde el borde o ribera del río $50 \mathrm{~m}$.

Estas zonas de protección serán de uso público, comunitario y de libre acceso. Se realizará el mantenimiento y conservación por el frentista. Las urbanizaciones del interés social a crearse a partir del presente PDOT estarán sujetas a reglamentación particular.

\section{Propuesta de lineamientos normativos de protección vial:}

Se formulan medidas de recuperación, para lo cual, en cumplimiento al Reglamento a la Ley Sistema Infraestructura Vial del Transporte Terrestre, art. 42 que establece de manera general que el derecho de vía se extenderá a 25 metros, medidos desde el eje de la vía hacia cada uno de los costados, distancia a partir de la cual podrá levantarse únicamente el cerramiento, debiendo para la construcción de vivienda observarse un retiro adicional de 5 metros. Para propuesta de nuevas vías rurales se establece 8 metros de ancho mínimo.

Propuesta de lineamientos normativos para suelo rural extractivo: Para determinar los lineamientos normativos de esta categoría de suelo, se realizó la identificación de la extracción de recursos naturales no renovables en base al catastro minero 2020; se ubica como polígonos de intervención únicamente los áridos y pétreos por ser de competencia municipal, por consideraciones de afección ambiental se determina restricciones absolutas de expansión a nuevas concesiones al tiempo de sugerir la no explotación minera y petrolera.

\section{Conclusiones:}

En los últimos cinco años, Palora enfrenta la expansión de la frontera agrícola con el cultivo 
de pitahaya, actividad productiva que se implanta sin restricción en todo el cantón incluyendo en las zonas periféricas al Parque Nacional Sangay. Sin duda, el alto lucro económico que representa hace atractiva esta actividad para quienes deseen emprender un sembrío y el capital determinará la extensión de la plantación. Lo anterior ha generado grandes conflictos y, sin duda, una posible solución se plantea en el presente estudio, dejando recomendaciones o lineamientos normativos para un uso del suelo rural, las que pueden convertirse en ordenanza. Cabe recordar que el presente estudio parte de la definición y ordenanza municipal que clasifica y delimita las áreas urbanas, fundamentalmente los centros poblados de las cuatro parroquias rurales y la cabecera cantonal Palora. Lo que lleva a definir como suelo rural el saldo territorial, es decir, aquel territorio que no es considerado urbano será clasificado como rural.

Dentro del suelo rural del cantón Palora se han establecido la sub-clasificación en cuatro tipos:

- Suelo rural de protección, mismo que contiene polígonos de intervención como áreas de riesgo. El patrimonio natural en estos polígonos plantea franjas de protección a los diferentes cuerpos de agua, cuyo ancho estará en función del lecho de cada río y también se ha generado lineamientos normativos estrictos para la protección vial en función de la jerarquización de estas.

- Suelo de producción: comprende polígonos de uso para actividad forestal, pastoril y sembríos determinando restricciones para el uso de un solo cultivo o monocultivo, con retiros y un máximo de aprovechamiento del lote en un $60 \%$ del área total, medida que limitará el uso de fertilizantes químicos.

- Suelo de aprovechamiento extractivo: se vinculó la información de todas actividades extractivas no renovables, cuya explotación es de materiales áridos, pétreos y también la minería no metálica siempre que tengan el derecho minero para su explotación. Se ha analizado la Ley de Minería para establecer el nivel de competencias de los GAD Municipales. Todas estas áreas extractivas se ubicaron en el territorio cantonal de Palora, para luego asignar la normativa prohibitiva de uso y gestión de suelo respectiva.

- Suelo de expansión urbana: se consideraron las áreas rurales periféricas a los asentamientos urbanos de todo el cantón Palora. El análisis de este tipo de suelo no existe, dado la poca densificación urbana. La capacidad de soporte del territorio y por la proximidad del Parque Sangay se ha generado una franja periférica al mismo, en la que no se podrá fraccionar los predios en menos de cinco hectáreas y donde estará restringido el uso absoluto de productos químicos en la producción agrícola.

\section{Referencias Bibliográficas}

Asamblea Nacional Constituyente. (2008). Constitución de la República del Ecuador. Registro Oficial 449 del 20 octubre de 2008. Quito, Ecuador.

Asamblea Nacional del Ecuador. (2016). Ley Orgánica de Ordenamiento Territorial, Uso y Gestión del Suelo de Ecuador. Oficio No. SAN-2016-1196. Quito, Ecuador.

Barroso, M., \& Flores, D. (2010). Teoría y estrategias de Desarrollo local. Universidad Internacional de Andalucía. https://dspace.unia.es/bitstream/handle/10334/3817/2010_desarrollolocal.pdf 
Consejo Técnico de Uso y Gestión del Suelo. (2020). Resolución N. 0005-CTUGS-2020. [Norma Técnica de Contenidos Mínimos, Procedimiento Básico de Aprobación y Proceso de Registro Formal de los Planes de Uso y Gestión de Suelo y Planes Urbanísticos Complementarios]. Quito, Ecuador.

Dollfus, O. (1997). La mundialización. Presses da Sciences Po.

Franco, J. (2013). Diseño de Políticas Públicas: Una Guía Práctica para Transformar Ideas en Proyectos Viables. IEXE Editorial.

Gehl, J. (2006). La humanización del espacio: La vida social entre los edificios. Reverte.

Gil, R., Boada, A., \& Alzate, I. (2018). Desarrollo endógeno y sustentable mediante un modelo de planificación estratégica. Caso de estudio: comuna Guaicamacuto, Venezuela. $\begin{array}{lll}\text { Revista } & \text { Espacios, } & \text { 39(32), }\end{array}$ https://www.revistaespacios.com/a18v39n32/a18v39n32p09.pdf

Gómez, D., \& Gómez, M. (2014). Marco conceptual para la ordenación territorial y reflexiones sobre el proceso ecuatoriano en la materia. IX Simposio nacional de desarrollo urbano y planificación territorial, http://www.sndu.org/ponencias/panel1/D_Gomez_Orea.pdf

Gómez, D., Ibagón, J., \& Forero, A. (2014). Desarrollo endógeno y latinoamérica. Revista Desarrollo $\quad y \quad$ Sociedad, $\quad 3(3), \quad 117-128$. https://jdc.edu.co/revistas/index.php/deyso/article/download/435/459/

Hoyuela, A., \& Citores, M. (2007). El desarrollo sostenible es un concepto relativo que depende del espacio, de la cultura y de la época (el tiempo).

Iturralde, C. (2019). Los paradigmas del desarrollo y su evolución: Del enfoque económico al multidisciplinario. Retos, 9(17), 7-23. doi:10.17163/ret.n17.2019.01

LOOTUGS, J. 2. (5 de julio de IV No790). Registro Oficial . Suplemento - Organo del Gobierno del Ecuador. Quito, Pichincha, Ecuador: Nacional.

Martínez-Echevarría, M. (2011). Técnica y crematística en Aristóteles. Revista empresa y humanismo, 14(2), 69-88. https://dadun.unav.edu/handle/10171/29136

Ministerio de Desarrollo Urbano y Vivienda. (s.f.). Dirección Nacional de Avalúos y Catastros (DINAC). Home: https://www.habitatyvivienda.gob.ec/direccion-nacional-de-avaluosy-catastros/

Organización de las Naciones Unidas para la Alimentación y la Agricultura [ONUAA]. (2018). Guía de buenas prácticas para la gestión y uso sostenible de los suelos en áreas rurales. Organización de las Naciones Unidas para la Alimentación y la Agricultura [ONUAA].

Pauta-Calle, F. (2019). ¿Son técnicamente viables los planes de uso y gestión de suelo previstos por la ley ecuatoriana de ordenamiento territorial? Nuevos paradigmas del espacio(13), 39-54. doi:10.29019/eidos.v13i1.543

Perroux, F. (1991). L'Economie du XXe siècle. Grenoble.

Plan de Desarrollo y Ordenamiento Territorial. (2015). https://issuu.com/hechosecuador/docs/canton_bolivar_actualizacion_pdyot_ 
Presidencia de la República del Ecuador. (2010). Código orgánico organización territorial autonomía descentralización. Quito, $\quad$ Ecuador. http://www.oas.org/juridico/pdfs/mesicic4_ecu_org.pdf

Presidencia de la República del Ecuador. (2010b). Código Orgánico de Planificación y Finanzas Públicas [COPYFP]. Quito, Ecuador.

Presidencia de la República del Ecuador. (2017). Código Orgánico del Ambiente. Quito, Ecuador.

Presidencia de la República del Ecuador. (2019). Decreto 680. Reglamento ley de ordenamieto territorial, uso y gestión de suelo. Quito, Ecuador.

Superintendencia de Uso y Gestión del Suelo [SOT]. (s.f.). Superintendencia de Uso y Gestión del Suelo [SOT]. https://www.sot.gob.ec/detalle/2/2/esp

Torres, V., Herrán, J., Narea, F., Montedeoca, L., Galárraga, L., Jaramillo, E., . . Benavides, J. (2011). Alternativas de vida: Trece experiencias de desarrollo endógeno en Ecuador. Universidad Politécnica Salesiana. https://dspace.ups.edu.ec/bitstream/123456789/6110/1/Alternativas\%20de\%20vida\% 20Trece\%20experiencias\%20de\%20desarrollo\%20endogeno\%20en\%20Ecuador.pdf

Valdizán, M. (2007). Universidad de Burgos y desarrollo local. Aproximación a un modelo teórico centrado en el desarrollo local desde la Universidad. Universidad de Burgos.

Vásquez, A. (2007). Desarrollo endógeno: Teorías y políticas de desarrollo territorial. Investigaciones regionales, 183-210.

Yépez, M. (2015). Los recursos naturales y el manejo de cuencas hidrográficas. Pontificia Universidad Católica del Ecuador. http://repositorio.puce.edu.ec/bitstream/handle/22000/8749/LOS\%20RECURSOS\%2 0NATURALES\%20Y\%20EL\%20MANEJO\%20DE\%20CUENCAS\%20HIDROGR $\%$ C3\%81FICAS.pdf?sequence $=1 \&$ is Allowed $=y$

\section{¿Ciencia}




\section{PARA CITAR EL ARTÍCULO INDEXADO.}

Quezada Ortega, V. P., Avila Calle, M. B., \& Castillo Ortega, Y. (2021). Propuesta de lineamientos normativos para el uso del suelo rural en el caso del cantón Palora - provincia de Morona Santiago . Ciencia Digital, 5(1), 114-132. https://doi.org/10.33262/cienciadigital.v5i1.1519

\section{¿Ciencia}

El artículo que se publica es de exclusiva responsabilidad de los autores y no necesariamente reflejan el pensamiento de la Revista Ciencia Digital.

El artículo queda en propiedad de la revista y, por tanto, su publicación parcial y/o total en otro medio tiene que ser autorizado por el director de la Revista Ciencia Digital.
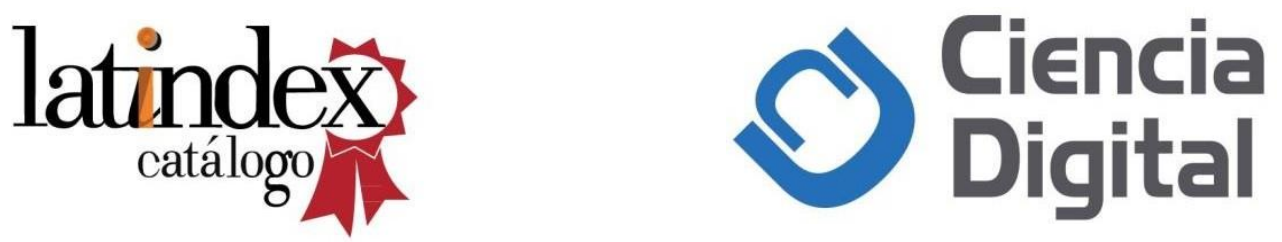